\title{
The Outcomes of Pediatric Hematopoietic Stem Cell Transplantation Recipients Requiring Intensive Care Unit Admission- A Single Center Experience
}

\author{
Royce Kwon, M.S. ${ }^{1,2}$, Sophia Koutsogiannaki, Ph.D. ${ }^{1,3}$, Steven J. Staffa, M.S. ${ }^{1,3}$ and Koichi Yuki, M.D. ${ }^{1,3^{*}}$ \\ ${ }^{1}$ Department of Anesthesiology, Critical Care and Pain Medicine, Cardiac Anesthesia Division, Boston Children's Hospital, \\ Boston, Massachusetts, USA
}

${ }^{2}$ Tufts University Faculty of Medicine, Boston, Massachusetts, USA

${ }^{3}$ Department of Anaesthesia, Harvard Medical School, Boston, Massachusetts, USA

\begin{abstract}
Background: Although the outcome of pediatric hematopoietic stem cell transplantation (HSCT) has significantly improved, it remains to be associated with high mortality. Identifying patients at high risk of mortality may potentially help to triage clinical management. The primary objective of this study is to evaluate risk factors associated with mortality of patients who received HSCT and admitted to ICU using pediatric sequential organ failure assessment (pSOFA), one of pediatric severity scoring systems in intensive care unit (ICU).
\end{abstract}

Methods: We performed retrospective review of electronic medical records of pediatric patients who received HSCT and were admitted to ICU in our institution between January 2010 and June 2018. Incidence of mortality was obtained, and risk factors associated with the mortality were examined using univariate and multivariable analyses.

Results: The mortality rate of pediatric HSCT patients who were admitted to ICU as a whole was $27.9 \%$. Patients were divided into three groups based on the number of HSCT required and timing of ICU admission. Patients who received first HSCT and admitted to ICU during the same hospital stay were the majority of the study population (Group A). $d(p S O F A)$, which was defined as the difference between maximum pSOFA and admission pSOFA, greater than and equal to 7 best predicted mortality of Group $A$ (the area under the ROC curve 0.850; $95 \% \mathrm{Cl}$ : 0.733-0.966). Univariate and multivariable analyses showed that an increase in neurologic and cardiovascular sub scores were independently associated with higher mortality (odds ratio (OR) 2.27; 95\% Cl: 1.32-3.93, and OR 2.69; 95\% Cl: 1.21-5.99, respectively).

Discussion: In our single center study, pediatric HSCT patients who were admitted to ICU demonstrated a high mortality. Risk factor analysis demonstrated that patients with the progression of neurologic and cardiovascular injuries probed by pSOFA scoring system during their ICU stay were strongly associated with mortality.

\section{Introduction}

Hematopoietic stem cell transplantation (HSCT) is instituted to treat a wide range of diseases including lymphoma, leukemia, immune-deficiency illness and myeloproliferative syndrome in pediatric population [1]. HSCT consists of intensive myeloablative chemoradio therapy followed by stem cell rescues. Stem cell rescues are accomplished with either autologous HSCT or allogeneic HSCT. With the improvement of conditioning regimens, human leukocyte antigen (HLA) typing, prevention and treatment of serious infections, transplantation outcome has improved significantly [1], and now its survival rate exceeds $80 \%[2,3]$. However, there is still anample room to improve the outcome. Knowing that the majority of patients who suffer from significant complications after HSCT are admitted to the intensive care unit (ICU) for treatment, it is critical to understand the characteristics of those patients and identify risk factors associated with poor outcomes among them.

A number of severity scoring systems have been established for patients who are admitted to ICU. They are largely divided into two systems [4]. One is the system based on the data on the first day of ICU admission. This includes acute physiology and chronic health evaluation (APACHE) scoring system, simplified acute physiology score (SAPS) and mortality prediction model (MPM). Another system is repetitive scoring system, which collects data sequentially throughout the duration of ICU stay or over the first few days, including sequential organ failure assessment (SOFA) and multiple organ dysfunction score (MODS). SOFA is a scoring system that assesses the performance of six organ systems in the body (neurologic, cardiovascular, respiratory, hepatic, renal and hematological systems) and assigns a score to each system (each organ score ranges from 0 to 4 , total 0-24). SOFA has been shown to be a powerful tool to predict mortality from hematologic malignancies [5]. Originally created for adults, pediatric SOFA version (pSOFA) incorporating age-adjusted scoring system of cardiovascular and renal systems was proposed [6]. Matics, et al. 
validated the PSOFA scoring system in the sepsis cohort. Using the pSOFA system, we examined risk factors of mortality of HSCT patients who were admitted to ICU in our institution.

\section{Methods}

\section{Data Collection}

After the Institutional Review Board (IRB) approval, data were retrospectively collected from the electronic medical record of pediatric patients (less than 18-yearsold) who were admitted to ICU with the diagnosis of HSCT from January 2010 to June 2018. Consent was waived by the IRB. We excluded patients who received the last HSCT before January 2010 or after June 2018, or who did not require admission to ICU following HSCT. We found 199 patients by initial search. Among them, 104 patients met the inclusion criteria. Patients were divided into three groups (A, B, and C) as follows; Group A- pediatric patients who were admitted to ICU after their first HSCT (75 patients). Group B-pediatric patients who received HSCT were discharged home but were readmitted to ICU due to post-transplant complications (15 patients). Group C- pediatric patients who had failed HSCT(s) were admitted to ICU after an additional HSCT (14 patients). The pSOFA scoring system proposed by Matics was used here to assess the degree of organ injury during the ICU stay.

\section{Statistical analysis}

Categorical variables were expressed as number and percentage, and continuous variables were expressed as median and interquartile range. Normality of continuous data was assessed using the Shapiro-Wilk test. Univariate analysis was done using the Mann-Whitney test or Student's t-test. For multivariable adjusted analysis, logistic regression modeling was performed. The results were presented as odds ratios as a measure of risk with accompanying $95 \%$ confidence intervals (C.I.). P values were obtained from the Wald test. Theoptimal cutoff value predicting mortality was derived from receiver operating characteristic (ROC) curve analysis by maximizing Youden's J index. Youden's J index is defined as J $=$ sensitivity + specificity -1 . The point in the ROC curve that maximizes the $J$ value is considered to be optimal for the cutoff point [7]. The statistical analyses were performed using PRISM software (GraphPad Software, San Diego, CA) and Stata 13 software (College Station, TX). A two-tailed $p<0.05$ was considered statistically significant. Assuming a Type I error rate of $5 \%$, our sample size of 55 survivors and 20 non-survivors in group $A$ resulted in $80 \%$ power for detecting a standardized difference (effect size) in d(pSOFA) values of 0.75 (average difference of 3 with standard deviation of 4 ) between the two groups, based on Student's t-test. Power analyses were performed using nQuery Advisor version 7.0 (Statistical Solutions Ltd., Cork, Ireland).

\section{Results}

\section{Demographics and pSOFA values of HSCT survivors and non-survivors who were admitted to ICU}

Figure 1 showed the number of survivors or nonsurvivors who required ICU admission. Survivors was defined as patients who were discharged from the hospital. Overall mortality was $27.9 \%$. The mortalities of patients in Group A, B and C were $26.7 \%, 26.7 \%$ and $53.7 \%$, respectively. Table 1 showed the characteristics of survivors and non-survivors in Group A, B and C. In Group $A$, non-survivors were older than survivors.

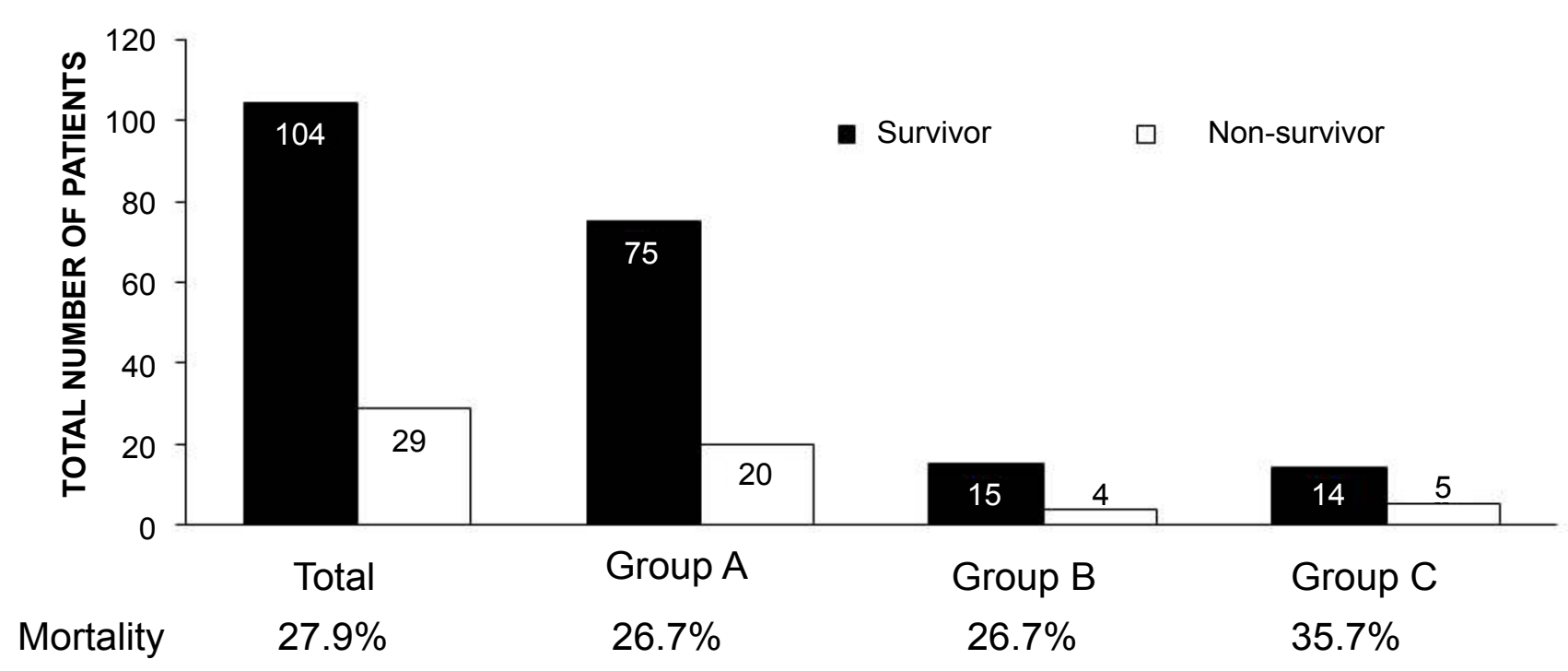

Figure 1: The number of patients who underwent pediatric hematologic stem cell transplantation during the study period in our institution.

The number of survivors and non-survivors and the percentages of mortality are shown. 
Table 1: Characteristics of patients who received HSCT and admitted to ICU

Profiles of survivors and non-survivors in Group A, B and C. Age, admission pSOFA, average pSOFA, maximum pSOFA and duration of ICU stay were shown as median $\left[25^{\text {th }}\right.$ percentile, $75^{\text {th }}$ percentile]. ${ }^{*}$ denotes statistical significance. C.I., confidence interval; n/a, not available.

Group A

\begin{tabular}{|l|l|l|l|l|}
\hline & Survivor $(\mathbf{n}=\mathbf{5 5})$ & Non-survivor $(\mathbf{n}=\mathbf{2 0})$ & P value & Odds ratio (95\% C.I.) \\
\hline Age (years) & $3.75[1.00,9.17]$ & $12.25[2.48,16.27]$ & $0.029^{*}$ & $1.09[1.01-1.17]$ \\
\hline Male Gender & $34(61.8 \%)$ & $9(45.0 \%)$ & 0.196 & $0.51[0.18-1.42]$ \\
\hline Admission pSOFA & $7.00[5.00,9.00]$ & $8.50[6.00,11.25]$ & 0.232 & $1.08[0.95-1.23]$ \\
\hline Average pSOFA & $6.00[5.19,7.85]$ & $12.70[10.14,15.67]$ & $<0.001^{*}$ & $2.23[1.49-3.33]$ \\
\hline Maximum pSOFA & $9.00[7.00,13.00]$ & $18.00[15.75,19.25]$ & $<0.001^{*}$ & $1.67[1.32-2.11]$ \\
\hline Duration of ICU stay & $11.00[5.00,32.00]$ & $33.50[11.00,51.50]$ & 0.446 & $1.01[0.99-1.02]$ \\
\hline
\end{tabular}

Group B

\begin{tabular}{|l|l|l|l|l|}
\hline & Survivor $(\mathbf{n}=\mathbf{1 1})$ & Non-survivor $(\mathbf{n}=\mathbf{4})$ & P value & Odds ratio (95\% C.I.) \\
\hline Age $($ years) & $7.33[2.25,9.75]$ & $14.58[13.46,15.27]$ & 0.113 & $1.24[0.95,1.62]$ \\
\hline Male Gender & $5(45.5 \%)$ & $0(0 \%)$ & n/a & n/a \\
\hline Admission pSOFA & $5.00[2.50,7.00]$ & $7.25[5.00,9.25]$ & 0.541 & $1.08[0.84,1.39]$ \\
\hline Average pSOFA & $3.75[2.89,5.83]$ & $8.60[7.85,9.12]$ & 0.074 & $1.78[0.95,3.35]$ \\
\hline Maximum pSOFA & $5.00[4.00,11.00]$ & $11.00[10.00,12.75]$ & 0.222 & $1.17[0.91,1.50]$ \\
\hline Duration of ICU stay & $4.00[1.00,5.00]$ & $4.00[4.00,31.50]$ & 0.307 & $1.04[0.97,1.11]$ \\
\hline
\end{tabular}

Group C

\begin{tabular}{|l|l|l|l|l|}
\hline & Survivor $(\mathbf{n}=\mathbf{9})$ & Non-survivor $(\mathbf{n}=\mathbf{5})$ & P value & Odds ratio (95\% C.I.) \\
\hline Age (years) & $6.42[2.58,13.42]$ & $5.50[2.42,8.92]$ & 0.428 & $0.92[0.74,1.33]$ \\
\hline Male Gender & $7(77.8 \%)$ & $4(80.0 \%)$ & 0.923 & $1.14[0.08,16.95]$ \\
\hline Admission pSOFA & $6.00[4.00,7.00]$ & $6.00[5.00,9.00]$ & 0.816 & $0.96[0.68,1.35]$ \\
\hline Average pSOFA & $6.21[5.33,7.75]$ & $12.31[8.69,14.83]$ & 0.111 & $1.29[0.99,1.68]$ \\
\hline Maximum pSOFA & $10.00[6.00,11.00]$ & $18.00[18.00,19.00]$ & 0.064 & $1.29[0.99,1.68]$ \\
\hline Duration of ICU stay & $9.00[7.00,11.00]$ & $29.00[21.00,63.00]$ & 0.167 & $1.03[0.99,1.07]$ \\
\hline
\end{tabular}

Table 2: Type of organ injury at the time of ICU admission

The frequency of each organ injury at the time of ICU was shown. Each organ injury was defined by pSOFA subscore >= 2 .

Group A

\begin{tabular}{|l|l|l|l|l|l|l|}
\hline & Neurologic & Cardiovascular & Respiratory & Hepatic & Renal & Hematologic \\
\hline Survivor & $23.6 \%$ & $21.8 \%$ & $45.5 \%$ & $20.0 \%$ & $16.4 \%$ & $92.7 \%$ \\
\hline Non-survivor & $20.0 \%$ & $35.0 \%$ & $65.0 \%$ & $30.0 \%$ & $20.0 \%$ & $95.0 \%$ \\
\hline
\end{tabular}

Group B

\begin{tabular}{|l|l|l|l|l|l|l|}
\hline & Neurologic & Cardiovascular & Respiratory & Hepatic & Renal & Hematologic \\
\hline Survivor & $18.2 \%$ & $27.3 \%$ & $36.4 \%$ & $9.1 \%$ & $18.2 \%$ & $54.5 \%$ \\
\hline Non-survivor & $50.0 \%$ & $25.0 \%$ & $75.0 \%$ & $50.0 \%$ & $0 \%$ & $50.0 \%$ \\
\hline
\end{tabular}

Group C

\begin{tabular}{|l|l|l|l|l|l|l|}
\hline & Neurologic & Cardiovascular & Respiratory & Hepatic & Renal & Hematologic \\
\hline Survivor & $22.2 \%$ & $0 \%$ & $22.2 \%$ & $33.3 \%$ & $0 \%$ & $88.9 \%$ \\
\hline Non-survivor & $0 \%$ & $0 \%$ & $40.0 \%$ & $20.0 \%$ & $20.0 \%$ & $80.0 \%$ \\
\hline
\end{tabular}

Average and maximum pSOFA values of non-survivors were significantly higher than those of survivors. In addition, the duration of ICU stay for non-survivors was significantly longer than that for survivors. In contrast, there was no statistical difference in age, pSOFA scores and duration of ICU stay between non-survivors and survivors in Group B and C. However, the median values of maximum and average pSOFA scores were higher 
and the median duration of ICU stay was longer in non-survivors, as in the case for Group A. The sample sizes of Group B and C were small, which may explain no statistical significance in these categories between survivors and non-survivors in Group B and C.

We defined injury of each organ system as pSOFA sub-score $>=2$ as previously described [8]. As expected, the frequency of hematologic injury at the time of ICU admission was higher in Group A and C, both of which represented patients who received HSCT and were admitted to ICU during the same hospital stay (Table 2).

\section{Table 3: Admission diagnosis to ICU}

Causes of admission to ICU were shown for each group. Number and percentage of patients are shown.

Group A

\begin{tabular}{|l|l|}
\hline Respiratory distress/failure & $60(80.0 \%)$ \\
Hemodynamic instability/Septic shock & $9(12.0 \%)$ \\
Others & $6(8.0 \%)$ \\
\hline
\end{tabular}

Group B

\begin{tabular}{|l|l|}
\hline Respiratory distress/failure & $12(80.0 \%)$ \\
Hemodynamic instability/Septic shock & $2(13.3 \%)$ \\
Others & $1(6.7 \%)$ \\
\hline
\end{tabular}

Group C

\begin{tabular}{|l|l|}
\hline Respiratory distress/failure & $12(85.8 \%)$ \\
Hemodynamic instability/Septic shock & $1(7.1 \%)$ \\
Others & $1(7.1 \%)$ \\
\hline
\end{tabular}

Table 4: The cutoff value of $d(p S O F A)$ to predict mortality $d(p S O F A)$ was defined as [maximum pSOFA - admission pSOFA]. The cutoff value of d(pSOFA) to best predict mortality was obtained from Youden-J index. C.I., confidence interval; AUC, area under the curve.

\begin{tabular}{|l|l|l|}
\hline Group & d(pSOFA) cut-off value & AUC [95\% C.I.] \\
\hline Group A & $=<7$ & $0.850[0.733-0.966]$ \\
\hline Group C & $=<8$ & $0.844[0.684-0.972]$ \\
\hline
\end{tabular}

In contrast, hematologic injury was seen in only a half of patients in Group B. Respiratory system was the second most injured organ at the time of admission (Table 2). The ICU admission diagnosis was listed in Table 3. Respiratory distress/failure was the major cause of ICU admission, which was in line with the data in Table 2.

\section{Admission pSOFA did not predict mortality but the larger difference between maximum pSOFA and admission pSOFA was associated with mortality}

Admission pSOFA between survivors and non-survivors did not show any difference in Group A-C, suggesting that this parameter would not serve to predict mortality (Table 1). Here we defined d(pSOFA) as [maximum pSOFA - admission pSOFA]. d(pSOFA) was compared between survivors and non-survivors in Group A-C (Figure 2). $d$ (pSOFA) was statistically larger in non-survivors than in survivors in Group A and C, but not in Group B. Based on this, d(pSOFA) was a better predictor than maximum $\mathrm{PSOFA}$ or average PSOFA.

Knowing that d(pSOFA) was larger in non-survivors than in non-survivors in Group A and C, we determined the cut-off value of $d$ (pSOFA) to predict mortality for both groups. The cutoff- value for Group A was equal to and above 7 (Table 4). The area under the curve (AUC) was 0.850 , which suggested that this cut-off value was a good predictor. The cutoff-value for Group $C$ was equal to and above 8 . The AUC was 0.844 .

\section{Type of organ injury associated with mortality}

Because pSOFA consists of six different domains, understanding the organ system succumbed to injury most would be helpful. Because a significant difference in $\mathrm{d}(\mathrm{p}$ SOFA) between survivors and non-survivors was noted in Group A and C, we examined the progression of each organ injury during ICU stay. We defined the difference in subscore of each organ at the time of admission and maximum pSOFA as $\mathrm{d}$ (subscore). $\mathrm{d}$ (subscore) was com-
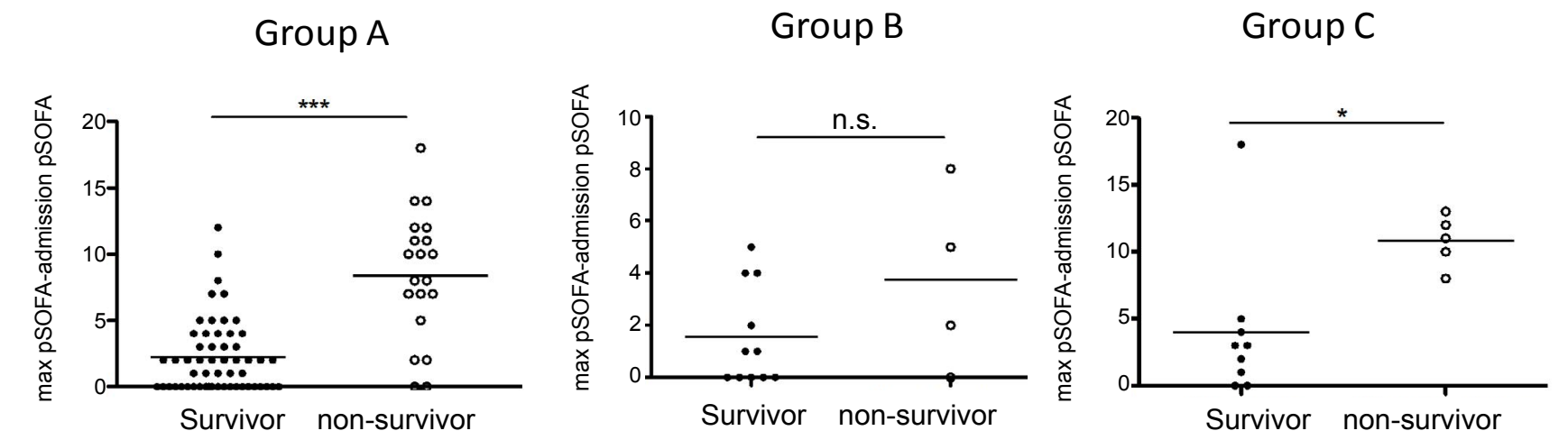

Figure 2: The comparison of difference between maximum pSOFA and admission pSOFA

Maximum pSOFA - admission pSOFA was defined as d(pSOFA). d(pSOFA) in Group A, B and C was compared between survivors and non-survivors. Statistical analysis was performed using Mann Whitney test. * and ${ }^{* *}$ denote $p<0.05$ and $p<$ 0.001 , respectively. n.s. $=$ not significant. 


\section{Table 5: Correlation between type of organ injury and mortality}

Correlation between progression of organ injury and mortality was examined using univariable and multivariable analyses for Group A and C. $d$ (subscore), defined as in the text, was used for this purpose and shown as median $\left[25^{\text {th }}\right.$ percentile, $75^{\text {th }}$ percentile] (mean). *denotes statistical significance. C.I., confidence interval.

Group A Univariate analysis

\begin{tabular}{|l|l|l|l|l|}
\hline Type of organ injury & Survivor $(\mathbf{n}=\mathbf{5 5})$ & Non-survivor $(\mathbf{n}=\mathbf{2 0})$ & P value & Odds ratio (95\% C.I.) \\
\hline Neurological injury & $0.00[0.00,1.00](0.64)$ & $4.00[3.00,4.00](3.00)$ & $<0.001^{*}$ & $2.92[1.86-4.58]$ \\
\hline Cardiovascular injury & $0.00[0.00,1.00](0.18)$ & $1.50[0.00,3.00](1.60)$ & $<0.001^{*}$ & $2.96[1.66-5.26]$ \\
\hline Respiratory injury & $0.00[0.00,0.00](0.65)$ & $1.50[0.25,2.75](1.55)$ & $0.010^{*}$ & $1.74[1.14-2.65]$ \\
\hline Hepatic injury & $0.00[0.00,0.00](0.31)$ & $2.00[0.00,2.00](1.20)$ & $0.003^{*}$ & $2.34[1.34-4.11]$ \\
\hline Renal injury & $0.00[0.00,1.00](0.32)$ & $1.00[0.00,2.00](1.00)$ & $0.019^{*}$ & $1.86[1.11-3.12]$ \\
\hline Hematologic injury & $0.00[0.00,0.00](0.09)$ & $0.00[-1.00,1.00](0.05)$ & 0.847 & $0.94[0.50-1.77]$ \\
\hline
\end{tabular}

Multivariable analysis

\begin{tabular}{|l|l|l|}
\hline Type of organ injury & P value & Odds ratio (95\% C.I.) \\
\hline Neurological injury & $0.003^{*}$ & $2.27[1.32-3.93]$ \\
\hline Cardiovascular injury & $0.015^{*}$ & $2.69[1.21-5.99]$ \\
\hline Respiratory injury & 0.117 & $0.57[0.28-1.15]$ \\
\hline Hepatic injury & 0.120 & $1.90[0.85-4.29]$ \\
\hline Renal injury & 0.122 & $2.01[0.83-4.85]$ \\
\hline
\end{tabular}

Group C Univariate analysis

\begin{tabular}{|l|l|l|l|l|}
\hline Type of organ injury & Survivor $(\mathbf{n = 9})$ & Non-survivor $(\mathbf{n = 5})$ & P value & Odds ratio (95\% C.I.) \\
\hline Neurological injury & $0.00[0.00,1.00](0.44)$ & $3.00[2.50,4.00](3.20)$ & n/a & n/a \\
\hline Cardiovascular injury & $0.00[0.00,2.00](0.78)$ & $3.00[1.00,3.50](2.40)$ & 0.100 & $1.98[0.88-4.48]$ \\
\hline Respiratory injury & $0.00[0.00,2.00](0.89)$ & $3.00[2.00,4.00](3.00)$ & 0.065 & $5.46[0.90-33.18]$ \\
\hline Hepatic injury & $0.00[0.00,1.50](0.78)$ & $1.00[0.00,2.00](1.00)$ & 0.738 & $1.17[0.47-2.88]$ \\
\hline Renal injury & $0.00[0.00,0.50](0.11)$ & $0.00[0.00,2.00](0.80)$ & 0.235 & $2.63[0.53-12.92]$ \\
\hline Hematologic injury & $1.00[0.00,1.00](1.00)$ & $0.00[-0.50,1.50](0.40)$ & 0.416 & $0.64[0.21-1.89]$ \\
\hline
\end{tabular}

pared for each organ system. In Group A, univariable analysis showed that neurologic, cardiovascular, respiratory, hepatic and renal $\mathrm{d}$ (subscore) were significantly higher in non-survivors. Multivariable analysis showed that only neurologic and cardiovascular d(subscore) were significantly higher in non-survivors. We also analyzed Group C similarly. We did not find any statistical significance between survivors and non-survivors. This could be due to a small sample size of Group C.

\section{Discussion}

Here we have shown that 1) pediatric HSCT recipients who required ICU admission demonstrated high mortality, and 2) patients requiring ICU admission after HSCT during the same hospital admission showed a strong correlation between their mortalities and $d(p-$ SOFA) scores. Multivariable analysis of Group A showed that neurologic and cardiovascular injuries had worsened significantly morein non-survivors than in survivors.

The pSOFA scores we used in this study was originally reported by Matics, et al. [6]. The cardiovascular and renal subscore system was modified to fit for different age group in pediatric population, and respiratory sub-score system incorporated oxygen saturation $\left(\mathrm{SpO}_{2}\right)$ /inspired oxygen concentration $\left(\mathrm{FiO}_{2}\right)$ in addition to partial pressure of oxygen $\left(\mathrm{PaO}_{2}\right) / \mathrm{FiO}_{2}$. The latter was introduced mainly due to unavailability of $\mathrm{PaO}_{2} / \mathrm{FiO}_{2}$ in a number of pediatric population. Two other pediatric versions of SOFA scoring system were proposed, but neither of them has not been validated yet $[9,10]$. The majority of HSCT patients are on anti-microbial medications. Thus with the exisitence of organ injury, technically some of them meet the criteria of sepsis. Because SOFA composes of current sepsis diagnosis criteria proposed in Sepsis-3 [11], we chose to test pSOFA here. Because admission PSOFA score was not a good predictor of survival in pediatric HSCT patients, we decided to examine the association between the mortality and the progression of organ injury during ICU stay. d(pSOFA) and d(subscore) could be good parameters to predict outcomes of pediatric HSCT patients. Although respiratory distress/ failure was common cause of admission to ICU, worsening neurologic or cardiovascular system was associated with mortality. However, this was a retrospective study in nature and we could not identify clear relationship 
between respiratory events and cardiovascular/neurological events. Identifying such causative relationship will be of significant help in clinical management.

Limitation of this study is as follows; this is a single center, retrospective study. Validation of utility of these parameters should be done in other institutions, possibly in the form of multi-center studies. Because of retrospective study in nature, there may be potential documentation errors in electronic medical record. Thus, validating this in prospective manner would be a next important step.

In conclusion, we found that $d(p S O F A)$ was strongly associated with mortality in pediatric patients who received HSCT and required ICU admission during the same admission. Subscore analysis showed that neurologic and cardiovascular injuries progressed more in non-survivors than in survivors.

\section{Financial Support}

This work was in part supported by CHMC Anesthesia Foundation (K.Y.), National Institute of Health R01GM118277 (K.Y.).

\section{Conflict of Interest}

None.

\section{References}

1. Hierlmeier S, Eyrich M, Wolfl M, Schlegel PG, Wiegering V. Early and late complications following hematopoietic stem cell transplantation in pediatric patients - A retrospective analysis over 11 years. PLoS One. 2018;13(10):e0204914. doi: 10.1371/journal.pone.0204914. PubMed PMID: 30325953; PubMed Central PMCID: PMCPMC6191171.

2. Duell $T$, van Lint MT, Ljungman $P$, Tichelli $A$, Socie $G$, Apperley JF, et al. Health and functional status of longterm survivors of bone marrow transplantation. EBMT Working Party on Late Effects and EULEP Study Group on Late Effects. European Group for Blood and Marrow Transplantation. Ann Intern Med. 1997;126(3):184-92. PubMed PMID: 9027268.

3. Hilgendorf I, Greinix H, Halter JP, Lawitschka A, Bertz H, Wolff $D$. Long-term follow-up after allogeneic stem cell transplantation. Dtsch Arztebl Int. 2015;112(4):51-8. doi: 10.3238/arztebl.2015.0051. PubMed PMID: 25797423; PubMed Central PMCID: PMCPMC4335490.

4. Bouch C, Thompson, JP. Severity scoring systems in the critically ill Continuing Education in Anaesthesia Critical Care \& Pain 2008;8(5):181-5.

5. Geerse DA, Span LF, Pinto-Sietsma SJ, van Mook WN Prognosis of patients with haematological malignancies admitted to the intensive care unit: Sequential Organ Failure Assessment (SOFA) trend is a powerful predictor of mortality. Eur J Intern Med. 2011;22(1):57-61. doi: 10.1016/j.ejim.2010.11.003. PubMed PMID: 21238895.

6. Matics TJ, Sanchez-Pinto LN. Adaptation and Validation of a Pediatric Sequential Organ Failure Assessment Score and Evaluation of the Sepsis-3 Definitions in Critically III Children. JAMA Pediatr. 2017;171(10):e172352. doi: 10.1001/jamapediatrics.2017.2352. PubMed PMID: 28783810 .
7. Zou KH, Yu CR, Liu K, Carlsson MO, Cabrera J. Optimal thresholds by maximizing or minimizing various metrics via ROC-type analysis. Acad Radiol. 2013;20(7):807-15. doi: 10.1016/j.acra.2013.02.004. PubMed PMID: 23582776.

8. Jacobson S, Liedgren E, Johansson G, Ferm M, Winso $O$. Sequential organ failure assessment (SOFA) scores differ between genders in a sepsis cohort: cause or effect? Ups J Med Sci. 2012;117(4):415-25. doi: 10.3109/03009734.2012.703255. PubMed PMID: 22793786; PubMed Central PMCID: PMCPMC3497227.

9. Shime N, Kawasaki T, Nakagawa S. Proposal of a New Pediatric Sequential Organ Failure Assessment Score for Possible Validation. Pediatr Crit Care Med. 2017;18(1):989. doi: 10.1097/PCC.0000000000001009. PubMed PMID: 28060166.

10. Schlapbach LJ, Straney L, Bellomo R, MacLaren G, Pilcher D. Prognostic accuracy of age-adapted SOFA, SIRS, PELOD-2, and qSOFA for in-hospital mortality among children with suspected infection admitted to the intensive care unit. Intensive Care Med. 2018;44(2):179-88. doi: 10.1007/ s00134-017-5021-8. PubMed PMID: 29256116; PubMed Central PMCID: PMCPMC5816088.

11. Singer M, Deutschman CS, Seymour CW, Shankar-Hari M, Annane D, Bauer M, et al. The Third International Consensus Definitions for Sepsis and Septic Shock (Sepsis-3). JAMA. 2016;315(8):801-10. doi: 10.1001/jama.2016.0287. PubMed PMID: 26903338; PubMed Central PMCID: PMCPMC4968574.

Corresponding Author: Koichi Yuki, M.D, Department of Anesthesiology, Critical Care and Pain Medicine, Cardiac Anesthesia Division, Boston Children's Hospital, 300 Longwood Avenue, Boston, Massachusetts, 02115, USA, Tel: 617-355-6225, Fax: 617-730-0894, E-mail: koichi. yuki@childrens.harvard.edu

Editor: Liming Zhang, MD, Department of Anesthesiology, University of Pittsburgh 200 Lothrop Street, UPMC MUH Suite N-467, Pittsburgh, PA 15213, United States of America; Tel: 412-648-6077; Email: zhangl1@anes. upmc.edu

\section{Additional publication details}

Journal short name: Transl Perioper \& Pain Med

Received Date: April 09, 2019

Accepted Date: May 19, 2019

Published Date: June 11, 2019

Citation: Kwon R, Koutsogiannaki S, Staffa SJ, Yuki K. The Outcomes of Pediatric Hematopoietic Stem Cell Transplantation Recipients Requiring Intensive Care Unit Admission- A Single Center Experience. Transl Perioper \& Pain Med 2019; 6(3):75-80

Copyright: (C) 2019 Kwon R, et al. This is an openaccess article distributed under the terms of the Creative Commons Attribution License, which permits unrestricted use, distribution, and reproduction in any medium, provided the original author and source are credited. 